(2) Points at Issue in the Chemotherapy of the Bacterial Infection in the Field of Internal Medicine

\author{
iii) Urinary Tract Infection
}

\author{
Atsushi Saito, M D \\ The Second Department of Medicine, Jikei University School of Medicine, Tokyo
}

Urinary tract infection is one of the most frequently encountered infectious diseases in the field of internal medicine and also is a general term for a wide variety of diseases ${ }^{11}$. Accordingly, chemotherapy for these diseases is by no means unitary and uniform, varying depending upon existing pathophysiology and stage of the disease.

In the present study we investigated the the following points at issue in the chemotherapy of urinary tract infection.

Isolation of causative organisms and their antibiotic sensitivity in urinary tract infection:

Causative organisms detected and the frequencies with which they were isolated in 44 cases of upper urinary tract infection and 61 cases of lower urinary tract infection where the diagnosis as to the site of infection (upper vs. lower) could be established by Fairley's method ${ }^{2)}$ or by examination of urine for antibody-coated bacteria ${ }^{3)}$ were: Escherichia coli, 63.6\% and 57.4\%; Klebsiella pneumoniae, $15.9 \%$ and 14.8\%; Pseudomonas aeruginosa, 9.1\% and 16.4\%; Proteus species, $6.8 \%$ and $8.2 \%$. There was no significant difference between upper and lower urinary tract infections in the frequency with which causative organisms were isolated. No significant difference seemed to exist between the two forms of urinary tract infection with respect to the antibiotic sensitivity pattern of isolated organisms.

\section{Pharmacokinetics of antibiotics as evaluated in relation to renal function and clinical application thereof :}

Pharmacokinetic studies were made of carbenicillin, cefazolin and tobramycin in 8 cases with normal renal function and 22 cases with impaired renal function.

In the presence of renal impairment carbenicillin, cefazolin and tobramycin all yielded long-sustained, high serum concentrations with proportionately low urinary concentrations and recovery rates. This tendency was particularly conspicuous with tobramycin. The relation of the elimination rate constant of these 3 antibiotics and creatinine clearance was given by the following equations:

carbenicillin $\cdots \cdots \mathrm{m}=0.00790 \mathrm{Ccr}+0.0369$

cefazolin $\quad \cdots \cdots \cdot \mathrm{m}=0.00489 \mathrm{Ccr}+0.0152$

tobramycin $\cdots \cdots \mathrm{m}=0.00371 \mathrm{Ccr}+0.00383$

Based on these pharmacokinetic features, the loading and maintenance doses for the drugs that can be used effectively and safely in patients with impaired renal function were estimated in accordance with the method of Dettli et al. and our proposed formula for the estimation of these dosage levels which embodies the patient's body weight and the dose per $\mathrm{kg}$ body weight as parameters (Table 1).

Table 1. Loading and maintenance dosage eqations of carbenicillin, cefazolin and tobramycin for patient with renal impairment.

\begin{tabular}{c|c|c}
\hline Antibiotics & \multicolumn{1}{|c|}{ Loading dose } & $\begin{array}{c}\text { Maintenance dose } \\
\text { (every 8 hours) }\end{array}$ \\
\hline $\mathrm{CBPC}$ & $\frac{\mathrm{D} \times \mathrm{W}}{5.2 \times 10^{4}}\left[(\mathrm{Ccr}+170)^{2}-20300\right]$ & $\frac{\mathrm{D} \times \mathrm{W}}{105}(\mathrm{Ccr}+4.7)$ \\
\hline $\mathrm{CEZ}$ & $\frac{\mathrm{D} \times \mathrm{W}}{4.7 \times 10^{4}}\left[(\mathrm{Ccr}+128)^{2}-3900\right]$ & $\frac{\mathrm{D} \times \mathrm{W}}{103}(\mathrm{Ccr}+3)$ \\
\hline TOB & $\frac{\mathrm{D} \times \mathrm{W}}{5.0 \times 10^{4}}\left[(\mathrm{Ccr}+129)^{2}-350\right]$ & $\frac{\mathrm{D} \times \mathrm{W}}{101}(\mathrm{Ccr}+1)$ \\
\hline
\end{tabular}

D: Dose in patient with normal renal function $(\mathrm{mg} / \mathrm{kg})$ W: Body weight $(\mathrm{kg})$ 
The method proposed by Dettli et al., ${ }^{4,5)}$ for the determination of optimal dosage of antibiotics has the disadvantage of requiring cumbersome calculations. In contrast, our own method can be used advantageously for the estimation of appropriate loading and maintenance doses of antibiotics permitting to dispense with any wearisome calculative steps.

\section{Significance of antibiotic concentrations in renal tissues on chemotherapeutic effect (upper urinary tract infection):}

In a study undertaken to verify the importance of antibiotic concentrations in renal tissues for chemotherapeutic effect in upper urinary tract infection, the relation between the concentration of cefazolin achieved in renal tissues and the effect of this antibiotic therapy was investigated in rats with experimental pyelonephritis induced by Proteus mirabilis (the minimum inhibitory concentration (MIC) of the antibiotic on this species being known to be $100 \mu \mathrm{g} / \mathrm{ml})$. The results indicated that whereas in a group of animals receiving $30 \mathrm{mg} / \mathrm{kg}$ cefazolin (a dose kown to produce antibiotic levels in renal tissues for exceeding its MIC on the causative organism) and excellent chemotherapeutic effect was obtained, little chemotherapeutic response was elicited in another group administered with $5 \mathrm{mg} / \mathrm{kg}$ cefazolin, a dose that fails to yield the MIC in affected tissues.

\section{Nephrotoxicity due to antibiotics:}

Many of antibiotics are excreted mainly by the kidney. These antibiotics yield exceedingly high concentrations in renal tissues and some of them even cause nephrotoxicity as a result of direct toxic effect.

Among nephrotoxic antibiotics, in particular, amphotericin B, polymyxin B and colistin are severe in toxicity, while the aminoglycosides and cephalosporins are relatively less toxic. Since, however, even antibiotics of the minimal nephrotoxic potentiality may prove to be more toxic in the presence of preexisting renal disease than otherwise, utmost care should be taken in performing chemotherapy in cases where there is preexisting renal disease, e.g. chronic pyelonephritis. Augmented nephrotoxicity due to the combined use of antibiotics or interactions between antibiotics and other drugs should also be cautioned against.

\section{Conclusions :}

1. There were no significant differences between upper and lower urinary tract infections in the frequency with which causative organisms were isolated as well as in the antibiotic sensitivity pattern. These findings are considered to suggest that upper urinary tract infection, in a greater majority of cases, might result, by ascending extension, from infection of the lower part of the urinary tract.

2. Based on the results of pharmacokinetic studies of carbenicillin, cefazolin and tobramycin, a method of using these 3 antibiotics in appropriate doses in the presence of impaired renal function was devised.

3. A study of chemotherapeutic results in upper urinary tract infection in relation to concentrations of antibiotics achieved in renal tissues showed that chemotherapeutic success with antibiotics depends to a larger extent upon their concentrations in renal tissues than upon the degree of sensitivity of causative organisms thereto.

4. In cases with preexisting renal disease, it is important to avoid the use of severely nephrotoxic antibiotics in the chemotherapy of upper urinary tract infection (esp. chronic stage).

Due attention should also be paid to the fact that the nephrotoxicity of an antibiotic might possibly be augmented by its interaction with another concomitant antibiotic or by its combined use with other types of drugs.

\section{REFERENCES}

1) Ueda Y : Pyelonephritis. J Jap Soc Intern Med 53 : 1231, 1965.

2) Fairley KF, et al: Simple test to determine the site of urinary tract infection. Lancet 2: 428, 1967.

3) Thomas U, et al: Antibody-coated bacteria in 
urine and site of urinary-tract infection. New Engl J Med 290: 588, 1974.

4) Dettli L, et al: Drug dosage in patients with impaired renal function. Postgrad Med J 46:
32, 1970.

5) Dettli L, et al: Multiple dose kinetics and drug dosage in patients with kidney disease. Acta Pharm Toxicol 29(Suppl) : 211, 1971.

\title{
(2) Points at Issue in the Chemotherapy of the Bacterial Infection in the Field of Internal Medicine
}

\section{iv) Septicemia in Elderly Patients}

\author{
Kaoru Shimada, M. D. \\ The Department of Internal Medicine, Tokyo Metropolitan Geriatric Hospital
}

\begin{abstract}
Over the past 30 years, the age distribution of bacteremia has changed markedly. The number of the patients with bacteremia between 10 and 40 years of age has dropped steadily while the population of those 70 years of age or older is increasing even more strikingly ${ }^{1}$. In this study, experiences with 210 bacteremic patients over 65 years of age are presented.
\end{abstract}

\section{MATERIAL AND METHOD}

Medical records of all patients with bacteremia in the Tokyo Metropolitan Geriatric Hospital between 1972 and 1975 were reviewed. In these patients, samples were obtained and inoculated at the bedside into culture bottle (Eiken) No. 1 (heart infusion broth), No. 2 (thioglycollate broth) and No. 3 (trypticase soy broth). Aerobic isolates were identified by standard procedure; obligate anaerobes were identified according to Finegold's method ${ }^{2)}$. Single isolation of Staph epidermidis, Corynebacterium or Propionibacterium from the blood culture was disregarded as a contaminant

\section{RESULTS}

(1) Incidence: The frequency of bacteremia per 100 hospital admission was 3.9 which is over three times the frequency of bacteremia in population in general hospitals. The frequency of bacteremia in patients who died during hospitalization per 100 hospital admissions was 13.3 episodes. The frequency of bacteremia in cancer patients who died during hospitalization was almost equal to that in patients who died of diseases other than cancer.

(2) Causative organisms (Table 1): Out of the 210 patients with bacteremia, more than one organisms were isolated from 37 patients. The total number of organisms isolated from the 210 patients was 265 . Out of these 265 organisms, 162 were aerobic gram negative rods (61.1\%), the majority being Enterobacteriaceae, 57 were aerobic gram positive organisms (21.5\%), the majority being Staph. aureus and enterococci, 40 were anaerobic bacteria (15.0\%) and 6 were fungi $(2.3 \%)$. It is interesting to note that the isolation of anaerobic bacteria in this series was much more frequent than any other previous reports. This may partially be related to the improved anaerobic technic we are using, and to the sepsis associated with decubital ulcers which occurs frequently in the aged patients with cerebral vascular lesions.

(3) Portal of entry of bacteria: The commonest portal of entry of bacteria in 210 patients was urinary tract (62 cases), followed by decubital ulcers (41), biliary tract (22), respiratory tract (14), intraabdominal infections other than biliary tract (12), soft tissue infections associated with venous cannulation (10), and others (3). The portal 